Submitted to the Annals of Statistics

\title{
A NOTE ON INSUFFICIENCY AND THE PRESERVATION OF FISHER INFORMATION
}

\author{
By David Pollard \\ Yale University
}

\begin{abstract}
Kagan and Shepp (2005) presented an elegant example of a mixture model for which an insufficient statistic preserves Fisher information. This note uses the regularity property of differentiability in quadratic mean to provide another explanation for the phenomenon they observed. Some connections with Le Cam's theory for convergence of experiments are noted.
\end{abstract}

1. Introduction. Suppose $\mathcal{P}=\left\{P_{\theta}: \theta \in \Theta\right\}$ is a statistical experiment, a set of probability measures on some measure space $(X, \mathcal{A})$ indexed by a subset $\Theta$ of the real line.

The Fisher information function $\mathbb{I}_{\mathcal{P}}(\theta)$ can be defined under various regularity conditions. If $S$ is a measurable map from $X$ into another measure space $\left(y, \mathcal{B}\right.$ ), each image measure $Q_{\theta}=S P_{\theta}$ (often called the distribution of $S$ under $P_{\theta}$, and sometimes denoted by $\left.P_{\theta} S^{-1}\right)$ is a probability measure on $\mathcal{B}$. The statistical experiment $\mathcal{Q}=\left\{Q_{\theta}: \theta \in \Theta\right\}$ is less informative, in the sense that an observation $y \sim Q_{\theta}$ tells us less about $\theta$ than an observation $x \sim P_{\theta}$. In particular, $\mathbb{I}_{\mathcal{Q}}(\theta) \leq \mathbb{I}_{\mathcal{P}}(\theta)$ for every $\theta$. If $S$ is a sufficient statistic the last inequality becomes an equality: there is no loss of Fisher information.

Statistical folklore holds that the converse is also true. For example, Lehmann and Casella (1998, page 158) set as an exercise the task of verifying, "under suitable regularity conditions", results stated by Basu (1964, Section 1), including the assertion that there is no loss of Fisher information if and only if the statistic is sufficient. They interpreted Basu's (unstated) regularity conditions to be "mainly concerned with interchange of integration with differentiation". Nevertheless, Kagan and Shepp (2005) (henceforth K\&S) were able to show, by means of a simple example, that it is possible to have $\mathbb{I}_{\mathcal{Q}}(\theta)=\mathbb{I}_{\mathcal{P}}(\theta)$ for every $\theta$ without $S$ being sufficient. The K\&S counterexample relies on another property - the support of a density

AMS 2000 subject classifications: Primary 60K35, 60K35; secondary 60K35

Keywords and phrases: Fisher information; sufficiency; Hellinger differentiability of probability models; differentiability in quadratic mean; score function; Le Cam's distance between statistical models

1

imsart-aos ver. 2011/11/15 file: pollard_april2012.tex date: May 25, 2018 
changing with the unknown parameter - that is notorious for upsetting classical statistical theory.

My purpose in this note is to make two small additions to the $\mathrm{K} \& \mathrm{~S}$ analysis.

(i) I reinterpret the phenomenon identified by $K \& S$, using the geometry of differentiabilty in quadratic mean.

(ii) Using an asymptotic argument, I offer an explanation for why the extent of the failure of sufficiency in the $K \& S$ example is too small to be captured by the Fisher information. More precisely, I explain why the experiment $Q_{n}$ obtained by $n$ independent replications of $Q$ is asymptotically equivalent (in Le Cam's sense) to the corresponding $\mathcal{P}_{n}$.

Most of the necessary theory is already available in the literature but is not widely known. The K\&S example provides a good showcase for that theory.

2. The K\&S example. What follows is a slightly simplified version of the K\&S construction.

Start from a smooth probability density

$$
g(w)=\frac{1}{2} w^{2} e^{-w}\{w>0\}
$$

with respect to Lebesgue measure $\mathfrak{m}$ on the real line. The power $w^{2}$ is chosen so that

$$
\frac{\dot{g}(w)^{2}}{g(w)}=g(w)\left(\frac{d \log g(w)}{d w}\right)^{2}=\frac{1}{2}(2-w)^{2} e^{-w}\{w>0\}
$$

is Lebesgue integrable. The shift family of densities $\{g(w-\theta): \theta \in \mathbb{R}\}$ has constant Fisher information,

$$
\mathbb{I}=\int_{-\infty}^{\infty} \dot{g}(w)^{2} / g(w) d w<\infty
$$

Let $\nu$ denote the probability measure that puts mass $1 / 2$ at each of +1 and -1 . For each $\theta \in \Theta=\mathbb{R}$ define a probability measure $P_{\theta}$ on (the Borel sigma-field of ) $X=\mathbb{R} \times\{-1,+1\}$ by means of its density

$$
f_{\theta}(x)=\{z=+1\} g(y-\theta)+\{z=-1\} g(\theta-y) \quad \text { where } x=(y, z) \in X
$$

with respect to the measure $\lambda:=\mathfrak{m} \otimes \nu$. That is, the coordinate $z$ has marginal distribution $\nu$ and the conditional distribution of $y$ given $z$ is that of $\theta+z w$ where $w \sim g$ independently of $z$.

imsart-aos ver. 2011/11/15 file: pollard_april2012.tex date: May 25, 2018 


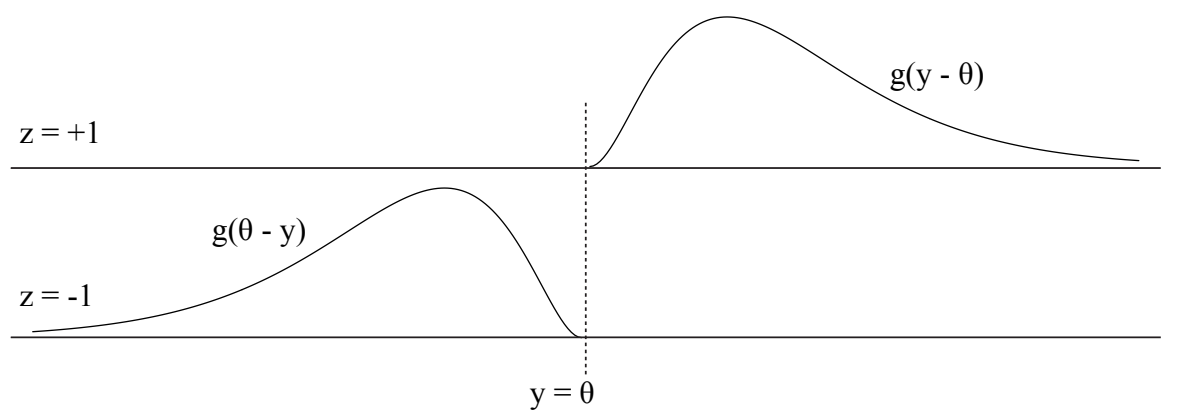

Here are the pertinent statistical facts for a single observation $x=(y, z)$ from $P_{\theta}$. (See Section 3 for some proofs.) Define $S(x)=y$ and $A(x)=z$. The marginal distribution $Q_{\theta}$ of $S$ has density

$$
h_{\theta}(y)=\frac{1}{2} g(y-\theta)+\frac{1}{2} g(\theta-y) \quad \text { with respect to } \mathfrak{m} .
$$

(a) The statistic $A$ is ancillary (its distribution, $\nu$, does not depend on $\theta$ ). By itself it gives no statistical information about $\theta$, but in conjunction with $S$ it does tell us something about $\theta$ : if $z=+1$ then (with $P_{\theta}$ probability one) $\theta<y$, and if $z=-1$ then $y<\theta$.

(b) The statistic $S$ is not sufficient because $\{z=+1\}=\{y>\theta\}$ a.s. $\left[P_{\theta}\right]$, implying $P_{\theta}(Z=+1 \mid y)=\{y>\theta\}$ almost surely. Equivalently,

$$
P_{\theta}(A(x)=1 \mid S)=\{S(x)>\theta\} \quad \text { a.s. }\left[P_{\theta}\right]
$$

which depends on $\theta$. (More formally, if $S$ were sufficient there would exist some measurable function $\pi(y)$ for which $P_{\theta}(z=1 \mid S)=\pi(S(x))$ a.s. $\left[P_{\theta}\right]$, for every $\theta$.)

(c) Both $\mathcal{P}:=\left\{P_{\theta}: \theta \in \mathbb{R}\right\}$ and $\mathcal{Q}:=\left\{Q_{\theta}: \theta \in \mathbb{R}\right\}$ have finite Fisher information: $\mathbb{I}_{\mathcal{Q}}(\theta)=\mathbb{I}_{\mathcal{P}}(\theta)=\mathbb{I}$ for all $\theta$, with $\mathbb{I}$ as in (1).

In short: There is no loss of Fisher information when only $S(x)$ is observed, even though $S$ is not a sufficient statistic.

Remark. K\&S used a slightly more involved construction, with density

$$
\begin{aligned}
f(x, \theta)= & \{z=+1\}[0.7 g(y-\theta)+0.3 g(\theta-y)] \\
& +\{z=-1\}[0.3 g(y-\theta)+0.7 g(\theta-y)] \quad \text { where } x=(y, z) \in X
\end{aligned}
$$

with respect to $\mathfrak{m} \otimes \mu$ where $\mu\{+1\}=\alpha=1-\mu\{-1\}$ and $\alpha \neq 1 / 2$. The analysis that I present can be extended to this $f_{\theta}$.

3. DQM interpretation. K\&S attributed the phenomenon in their version of the example in Section 2 to a failure of strict convexity of Fisher

imsart-aos ver. 2011/11/15 file: pollard_april2012.tex date: May 25, 2018 
information with respect to mixtures of statistical experiments. There is another explanation involving the geometry of Hellinger derivatives, which I find more illuminating.

By a theorem of Hájek (1972, Lemma A.3), Lebesgue integrability of the function $\dot{g}^{2} / g$ in (1) implies that the set of densities $\mathcal{G}:=\{g(y-\theta): \theta \in \mathbb{R}\}$ (with respect to Lebesgue measure) is Hellinger differentiable with Hellinger derivative $\gamma(y-\theta)$ at $\theta$, where

$$
\gamma(w):=\frac{-\dot{g}(w)}{2 \sqrt{g(w)}}=\frac{(2-w)}{2 \sqrt{2}} e^{-w / 2}\{w>0\} .
$$

That is,

$$
\int|\sqrt{g(y-\theta-t)}-\sqrt{g(y-\theta)}-t \gamma(y-\theta)|^{2} d y=o\left(t^{2}\right) \quad \text { as } t \rightarrow 0 .
$$

This assertion is also easy to check by explicit calculations. (See Lehmann and Romano (2005, Cor. 12.2.1) for details.)

The family of densities $\mathcal{F}:=\left\{f_{\theta}(x): \theta \in \mathbb{R}\right\}$, for $f_{\theta}$ as in (2), inherits the Hellinger differentiability from $\mathcal{G}$ :

$$
\int\left|\sqrt{f_{\theta+t}(x)}-\sqrt{f_{\theta}(x)}-t \zeta_{\theta}(x)\right|^{2} \lambda(d x)=o\left(t^{2}\right) \quad \text { as } t \rightarrow 0,
$$

for the Hellinger derviative

$$
\zeta_{\theta}(x):=\{z=+1\} \gamma(y-\theta)-\{z=-1\} \gamma(\theta-y) .
$$

The significance of approximation (3) becomes clearer when it is rewritten as a differentiability property of the likelihood ratios. That is, it helps to work with the square root of the density of $P_{\theta+t}$ with respect to $P_{\theta}$. Unfortunately, $P_{\theta+t}$ is not dominated by $P_{\theta}$. In general, to eliminate such an embarrassment one needs to split $P_{\theta+t}$ into a singular part $P_{t, \theta}^{\perp}$, which concentrates on a set of zero $P_{\theta}$ measure, plus a part $P_{\theta+t}^{(a b s)}$ that has a density $p_{t, \theta}$ with respect to $P_{\theta}$. For reasons related to the asymptotic theory for repeated sampling, it is customary to make a small extra assumption about the behavior of $P_{t, \theta}^{\perp} X$ as $t$ tends to zero. Following Le Cam (1986, Section 17.3) and Le Cam and Yang (2000, Section 7.2), I will call the slightly stronger property differentiability in quadratic mean (DQM), to stress that the definition requires a little more than Hellinger differentiability.

Remark. Some authors (for example, Bickel et al. 1993, page 457) use the term DQM as a synonym for Hellinger differentiability.

imsart-aos ver. 2011/11/15 file: pollard_april2012.tex date: May 25, 2018 
Definition 4. Say that $\mathcal{P}=\left\{P_{\theta}: \theta \in \Theta\right\}$, with $\Theta \subseteq \mathbb{R}$, is differentiable in quadratic mean (DQM) at $\theta$ with score function $\Delta_{\theta}(x)$ if, for $\theta+t \in \Theta$,

(i) for the part $P_{t, \theta}^{\perp}$ of $P_{\theta+t}$ that is singular with respect to $P_{\theta}$,

$$
P_{t, \theta}^{\perp}(X)=o\left(t^{2}\right) \quad \text { as } t \rightarrow 0
$$

(ii) $\Delta_{\theta} \in \mathcal{L}^{2}\left(P_{\theta}\right)$

(iii) the absolutely continuous part of $P_{\theta+t}$ has density $p_{t, \theta}(x)$ with respect to $P_{\theta}$ for which

$$
\sqrt{p_{t, \theta}(x)}=1+\frac{1}{2} t \Delta_{\theta}(x)+r_{t, \theta}(x) \quad \text { with } P_{\theta}\left(r_{t, \theta}^{2}\right)=o\left(t^{2}\right) \text { as } t \rightarrow 0 .
$$

Call $\mathcal{P} D Q M$ if it is $D Q M$ at each $\theta$ in $\Theta$.

Remark. The factor of $1 / 2$ in requirement (iii) ensures that $P_{\theta} \Delta_{\theta}^{2}$ is equal to the Fisher information $\mathbb{I}_{\mathcal{P}}(\theta)$ if the densities are suitably smooth in a pointwise sense.

The $\mathcal{P}$ from Section 2 is, in fact, DQM. For $t>0$ the singular part $P_{t, \theta}^{\perp}$ has density $\{z=-1\} g(\theta-y)\{\theta<y<\theta+t\}$ with respect to $\lambda$, so that $P_{t, \theta}^{\perp}(X)=O\left(|t|^{3}\right)$. The part of $P_{\theta+t}$ that is dominated by $P_{\theta}$ has density

$$
\begin{aligned}
& p_{t, \theta}(x)=\frac{f_{\theta+t}(x)}{f_{\theta}(x)}\left\{f_{\theta}(x)>0\right\} \\
&5) \quad=\{z=+1\} \frac{g(y-\theta-t)}{g(y-\theta)}\{y>\theta\}+\{z=-1\} \frac{g(\theta+t-y)}{g(\theta-y)}\{y<\theta\} .
\end{aligned}
$$

There is a similar expression for the case $t<0$. The score function equals

$$
\begin{aligned}
\Delta_{\theta}(x) & =2 \frac{\zeta_{\theta}(x)}{\sqrt{f_{\theta}(x)}}\left\{f_{\theta}(x)>0\right\} \\
& =\{z=+1\} \frac{\gamma(y-\theta)}{\sqrt{g(y-\theta)}}\{y>\theta\}-\{z=-1\} \frac{\gamma(\theta-y)}{\sqrt{g(\theta-y)}}\{y<\theta\} .
\end{aligned}
$$

The density $p_{t, \theta}$ and the score function $\Delta_{\theta}(x)$ are uniquely determined only up to a $P_{\theta}$ equivalence. As noted for fact (b) near the end of Section 2, sufficiency fails for $S$ because

$$
\{z=+1\}=\{y>\theta\} \quad \text { a.s. }\left[P_{\theta}\right]
$$

Similarly $\{z=-1\}=\{y<\theta\}$ a.s. $\left[P_{\theta}\right]$. Together these two equivalences explain why no Fisher information is lost. The score function $\Delta_{\theta}$ is changed 
only on a $P_{\theta}$-negligible set if we omit the two indicator functions involving $z$ from (6). In effect, the score function $\Delta_{\theta}(x)$ depends on $x$ only through the value of the statistic $S$. As the next theorem (which is proved in Section 5) shows, that property is equivalent to the preservation of Fisher information.

TheOrem 7. Suppose $\mathcal{P}=\left\{P_{\theta}: \theta \in \Theta\right\}$ on $(X, \mathcal{A})$ is DQM with score function $\Delta_{\theta}$. Suppose $S$ is a measurable map from $(\mathcal{X}, \mathcal{A})$ into $(\mathcal{y}, \mathcal{B})$ and $Q_{\theta}=S P_{\theta}$ is the distribution of $S$ under $P_{\theta}$. Then:

(i) The statistical experiment $\mathcal{Q}=\left\{Q_{\theta}: \theta \in \Theta\right\}$ is also $D Q M$, with score function $\widetilde{\Delta}_{\theta}(y)=P_{\theta}\left(\Delta_{\theta} \mid S=y\right)$.

(ii) At each fixed $\theta$, Fisher information is preserved (that is, $\mathbb{I}_{\mathcal{P}}(\theta)=\mathbb{I}_{Q}(\theta)$ ) if and only if $\Delta_{\theta}(x)=\widetilde{\Delta}_{\theta}(S x)$ a.s. $\left[P_{\theta}\right]$.

With only notational changes, the Theorem extends to the case where $\Theta$ is a subset of some Euclidean space; no extra conceptual difficulties arise in higher dimensions.

Credit where credit is due. The results stated in Theorem 7 have an interesting history. Property (i) was asserted ("Direct calculations show that the function $q^{1 / 2}(y ; \theta)$ is differentiable in $L_{2}(\widetilde{\nu})$ and possess a continuous derivative ...") in Theorem 7.2 of Ibragimov and Has'minskii (1981, Chapter I, page 70), an English translation from the 1979 Russian edition. However, that Theorem also (incorrectly, as noted by K\&S) asserted that Fisher information is preserved if and only if $S$ is sufficient.

Pitman (1979, pages 19-21) established differentiability in mean, a property slightly different from (i), in order to deduce a result equivalent to (ii).

Le Cam and Yang (1988, Section 7) deduced an analogue of (i) (preservation of DQM under restriction to sub-sigma-fields) by an indirect argument using equivalence of DQM with the existence of a quadratic approximation to likelihood ratios of product measures (an LAN condition).

Bickel et al. (1993, page 461) proved result (i), citing Ibragimov and Has'minskii (1981), Le Cam and Yang (1988), and van der Vaart (1988, Appendix A3) for earlier proofs. The last of these was a revised ("I have not resisted the temptation to rewrite numerous parts of the original manuscript") version of van der Vaart's 1987 Ph.D. thesis. He cited Le Cam and Yang (1988) and a manuscript version of Bickel et al. (1993).

4. Large sample interpretation. The example in Section 2 shows that, for a sample $x=(y, z)$ of size one from $P_{\theta}$, some "statistical information about $\theta$ (namely, whether $\theta<y$ or $\theta>y$ ) is lost if we discard $z$. The loss is not detected by Fisher's measure of information. An asymptotic

imsart-aos ver. 2011/11/15 file: pollard_april2012.tex date: May 25, 2018 
analysis, based on a sample of size $n$ from $P_{\theta}$, sheds a little light on why the $z$ contribution is relatively unimportant.

Write $\mathbb{P}_{\theta, n}$ and $\mathbb{Q}_{\theta, n}$ for the $n$-fold product measures $P_{\theta}^{n}$ and $Q_{\theta}^{n}$, with the probability measures $P_{\theta}$ and $Q_{\theta}$ as in Section 2 . That is, the statistical experiment $\mathcal{P}_{n}=\left\{\mathbb{P}_{\theta, n}: \theta \in \Theta\right\}$ corresponds to taking $n$ independent observations $x_{1}=\left(y_{1}, z_{1}\right), \ldots, x_{n}=\left(y_{n}, z_{n}\right)$ from $P_{\theta}$ and $Q_{n}=\left\{\mathbb{Q}_{\theta, n}: \theta \in \Theta\right\}$ corresponds to $y_{1}, \ldots, y_{n}$.

Both $\mathcal{P}_{n}$ and $Q_{n}$ are locally asymptotically normal (Le Cam and Yang, 2000, Chapter 6). They share the same local normal approximations because that have the same score functions and (hence) the same Fisher information functions: for each fixed $\theta$ and each finite subset $T$ of the real line, the "local experiments"

$$
\left\{\mathbb{P}_{\theta+t n^{-1 / 2}, n}: t \in T\right\} \quad \text { and } \quad\left\{\mathbb{Q}_{\theta+t n^{-1 / 2}, n}: t \in T\right\}
$$

are asymptotically equivalent in the (weak) Le Cam sense. The deficiency distance (Le Cam and Yang, 2000, Section 2.2) between these two local experiments tends to zero as $n$ tends to infinity. The local asymptotic equivalence of $\mathcal{P}_{n}$ and $\mathcal{Q}_{n}$ has many consequences. For example, classical theory establishes existence of many different estimators $\widehat{\theta}_{n}=\widehat{\theta}_{n}\left(x_{1}, \ldots, x_{n}\right)$ for which $\sqrt{n}\left(\widehat{\theta}_{n}-\theta\right)$ converges in distribution under $\mathbb{P}_{\theta, n}$ to $N\left(0, \mathbb{I}^{-1}\right)$, and many different estimators $\theta_{n}^{*}=\theta_{n}^{*}\left(y_{1}, \ldots, y_{n}\right)$ for which $\sqrt{n}\left(\theta_{n}^{*}-\theta\right)$ converges in distribution under $\mathbb{Q}_{\theta, n}$ to the same $N\left(0, \mathbb{I}^{-1}\right)$. As shown by the Hájek-Le Cam convolution and asymptotic minimax theorems (Bickel et al., 1993, Section 2.3), there are various senses in which the $N\left(0, \mathbb{I}^{-1}\right)$ limit is the best we can hope to achieve for either experiment. Asymptotically speaking, the $z_{i}$ 's must be contributing at a less important level.

Remark. Except for the purpose of the root- $n$ asymptotics, perhaps we should agree with Basu (1975, Section 5) that the Fisher information function is a "mathematically interesting but statistically rather fruitless notion".

For $i=1, \ldots, n$, write $y_{L: n}$ for the largest $y_{i}$ for which $z_{i}=-1$ and $y_{R: n}$ the smallest $y_{i}$ for which $y_{i}=+1$. Each $z_{i}$ tells us whether $y_{i}>\theta$ or $y_{i}<\theta$, implying

$$
y_{L: n}<\theta<y_{R: n} \quad \text { with } \mathbb{P}_{\theta, n} \text { probability one. }
$$

The $w^{2}$ decay in $g(w)$ at zero, implies that both $\theta-y_{L: n}$ and $y_{R: n}-\theta$ are decreasing at an $n^{-1 / 3}$ rate. In fact both $n^{1 / 3}\left(\theta-y_{L: n}\right)$ and $n^{1 / 3}\left(y_{R: n}-\theta\right)$ have nontrivial limit distributions under $\mathbb{P}_{\theta, n}$. For example, for each $s>0$

imsart-aos ver. 2011/11/15 file: pollard_april2012.tex date: May 25, 2018 
direct calculation shows that $P_{\theta}\left(\theta, \theta+s n^{-1 / 3}\right)=s^{3} /(6 n)+o(1 / n)$, so that $\mathbb{P}_{\theta, n}\left\{n^{1 / 3}\left(y_{R: n}-\theta\right)>s\right\}=\mathbb{P}_{\theta, n}\left\{\right.$ no $z_{i}{ }^{\prime}$ s in $\left.\left(\theta, \theta+s n^{-1 / 2}\right)\right\} \rightarrow \exp \left(-s^{3} / 6\right)$.

For any $n^{-1 / 2}$-consistent estimator $\widehat{\theta}_{n}$ the event $A_{n}=\left\{y_{L: n}<\widehat{\theta}_{n}<y_{R: n}\right\}$ has $\mathbb{P}_{\theta, n}$ probability that tends very rapidly to one. Put another way,

$$
\mathbb{P}_{\theta, n}\left\{\exists i \leq n: \theta<y_{i}<\widehat{\theta}_{n} \text { or } \widehat{\theta}_{n}<y_{i}<\theta\right\} \rightarrow 0 .
$$

With high probability, what we learn from the $z_{i}$ 's just duplicates what we usually can learn from the $y_{i}$ 's.

To make the idea more concrete, define $z_{i, n}^{*}=\operatorname{sgn}\left(y_{i}-\widehat{\theta}_{n}\right)$ and $x_{i, n}^{*}=$ $\left(y_{i}, z_{i, n}^{*}\right)$. That is,

$$
z_{i, n}^{*}=\left\{\begin{array}{ll}
+1 & \text { if } y_{i}>\widehat{\theta}_{n} \\
-1 & \text { if } y_{i}<\widehat{\theta}_{n}
\end{array} .\right.
$$

On the event $A_{n}$ we have $x_{i}=x_{i, n}^{*}$ for $i=1, \ldots, n$. If $\mathbb{P}_{\theta, n}^{*}$ denotes the joint distribution of $x_{1, n}^{*}, \ldots, x_{n, n}^{*}$ then

$$
\sup _{\theta \in \Theta}\left\|\mathbb{P}_{\theta, n}^{*}-\mathbb{P}_{\theta, n}\right\|_{\mathrm{TV}} \leq \sup _{\theta \in \Theta} \mathbb{P}_{\theta, n} A_{n}^{c} \rightarrow 0
$$

In the terminology of Le Cam's theory for convergence of statistical experiments, $\mathcal{P}_{n}$ and $\mathcal{Q}_{n}$ are asymptotically equivalent, not just locally asymptotically equivalent in the weak sense. The vector $\left(y_{1}, \ldots, y_{n}\right)$ is asymptotically sufficient for $\mathcal{P}_{n}$, in Le Cam's sense. The map $\left(y_{1}, \ldots, y_{n}\right) \mapsto\left(x_{1, n}^{*}, \ldots, x_{n, n}^{*}\right)$ defines a Le Cam transition (Le Cam and Yang, 2000, Theorem 2.2) that bounds the deficiency $\delta\left(Q_{n}, \mathcal{P}_{n}\right)$.

Put another way, for every statistic $\psi_{n}\left(x_{1}, \ldots, x_{n}\right)$ for $\mathcal{P}_{n}$ there is another statistic $\psi_{n}^{*}\left(y_{1}, \ldots, y_{n}\right)=\psi_{n}\left(x_{1, n}^{*}, \ldots, x_{n, n}^{*}\right)$ for $Q_{n}$ that has the same asymptotic behavior.

Remark. Rough calculations suggest that the Le Cam distance between $\mathcal{P}_{n}$ and $Q_{n}$ tends to zero like $\exp \left(-C n^{1 / 3}\right)$ for some constant $C$. I omit the details because the actual rate is not important for the story I am telling.

5. Proof of Theorem 7. Recall that the Kolmogorov conditional expectation $P_{\theta}(\cdot \mid S=y)$ is abstractly defined, via the Radon-Nikodym theorem, as an increasing linear map (depending on $\theta) \kappa: L^{1}\left(P_{\theta}\right) \rightarrow L^{1}\left(Q_{\theta}\right)$ with properties analogous to those enjoyed by a Markov kernel. If we identify an $f$ in $L^{1}\left(P_{\theta}\right)$ with the (signed) measure $\mu_{f}$ for which $d \mu_{f} / d P_{\theta}=f$, then $g=\kappa f$ is the density of $S \mu_{f}$ with respect to $Q_{\theta}$. To stress the analogy

imsart-aos ver. 2011/11/15 file: pollard_april2012.tex date: May 25, 2018 
with Markov kernels I will write $\kappa_{y} f$, or even $\kappa_{y} f(x)$, instead of $(\kappa f)(y)$. Thus the defining property of $\kappa$ can be rewritten as

$$
Q_{\theta} f_{1}(y) \kappa_{y} f_{2}=P_{\theta} f_{1}(S x) f_{2}(x)
$$

for measurable real functions $f_{1}$ on $y$ and $f_{2}$ on $X$, at least when $f_{1}(S x) f_{2}(x)$ is $P_{\theta}$-integrable. A reader who chose to interpret $\kappa_{y}$ as a Markov kernel would lose only a tiny amount of generality.

Of course if one regards $\kappa$ as acting on the function $\mathcal{L}^{1}\left(P_{\theta}\right)$, instead of on the space $L^{1}\left(P_{\theta}\right)$ of $P_{\theta}$-equivalence classes, then one should qualify assertions with the occasional a.s. $\left[P_{\theta}\right]$ caveats and regard $\kappa f$ as being defined only up to $Q_{\theta}$ equivalence. Following the usual custom, I will omit such qualifiers.

Proof of assertion (i). The following argument is adapted from van der Vaart (1988, Appendix A3).

To simplify notation, I will prove that $Q$ is DQM only at $\theta=0$, writing $P_{t}^{\perp}$ instead of $P_{t, 0}^{\perp}$ and $p_{t}$ instead of $p_{t, 0}$. Keep in mind that $\kappa_{y}$ now denotes the conditional expectation operator $P_{0}(\cdot \mid S=y)$. For each function $h(x)$ in $\mathcal{L}^{2}\left(P_{0}\right)$ I will write $\widetilde{h}(y)$ for its conditional mean $\kappa_{y} h(x)$ and

$$
\operatorname{var}_{y} h:=\kappa_{y}(h(x)-\widetilde{h}(y))^{2}=\kappa_{y} h(x)^{2}-\widetilde{h}(y)^{2}
$$

for its conditional variance.

Start with the simplest case where $P_{t}$ is actually dominated by $P_{0}$. Then

$$
\xi_{t}(x)=\sqrt{d P_{t} / d P_{0}}=1+\frac{1}{2} t \Delta_{0}(x)+r_{t}(x) \quad \text { with } P_{0} r_{t}^{2}=o\left(t^{2}\right)
$$

and

(10) $\widetilde{\xi}_{t}(y):=\kappa_{y} \xi_{t}(x)=1+\frac{1}{2} t \widetilde{\Delta}_{0}(y)+\widetilde{r}_{t}(y) \quad$ with $Q_{0} \widetilde{r}_{t}^{2} \leq P_{0} r_{t}^{2}=o\left(t^{2}\right)$.

and, by the Radon-Nikodym property,

$$
\eta_{t}(y)=\sqrt{d Q_{t} / d Q_{0}}=\sqrt{\kappa_{y} \xi_{t}(x)^{2}}
$$

The proof of assertion (i) will work by showing that the difference $\delta_{t}(y):=$ $\eta_{t}(y)-\widetilde{\xi}_{t}(y)$ is small, in the sense that $Q_{0} \delta_{t}^{2}=o\left(t^{2}\right)$. For then we will have

$$
\eta_{t}(y)=1+\frac{1}{2} t \widetilde{\Delta}_{0}(y)+\left[\widetilde{r}_{t}(y)+\delta_{t}(y)\right] \quad \text { with } Q_{0}\left[\widetilde{r}_{t}(y)+\delta_{t}(y)\right]^{2}=o\left(t^{2}\right),
$$

which implies DQM for $\mathcal{Q}$ at 0 .

The desired property for $\delta_{t}$ will be derived from the following three facts about the conditional variance

$$
\sigma_{t}^{2}(y):=\operatorname{var}_{y}\left(\xi_{t}\right)=\kappa_{y} \xi_{t}(x)^{2}-\widetilde{\xi}_{t}(y)^{2}=\eta_{t}(y)^{2}-\widetilde{\xi}_{t}(y)^{2} .
$$

imsart-aos ver. 2011/11/15 file: pollard_april2012.tex date: May 25, 2018 
(a) The representation $\sigma_{t}^{2}(y)=\kappa_{y}\left(\xi_{t}(x)-\widetilde{\xi}_{t}(y)\right)^{2}$ gives

$$
\begin{aligned}
\sigma_{t}^{2}(y) & =\kappa_{y}\left(\frac{1}{2} t\left[\Delta_{0}(x)-\widetilde{\Delta}_{0}(y)\right]+\left[r_{t}(x)-\widetilde{r}_{t}(y)\right]\right)^{2} \\
& \leq 2\left(\frac{1}{2} t\right)^{2} \kappa_{y}\left[\Delta_{0}(x)-\widetilde{\Delta}_{0}(y)\right]^{2}+2 \kappa_{y}\left[r_{t}(x)-\widetilde{r}_{t}(y)\right]^{2} \\
& \leq \frac{1}{2} t^{2} \kappa_{y} \Delta_{0}^{2}+2 \kappa_{y} r_{t}^{2} .
\end{aligned}
$$

Remark. The cancellation of the leading 1 when $\widetilde{\xi}_{t}$ is subtracted from $\xi_{t}$ seems to be vital to the proof. For general Hellinger differentiability, the cancellation would not occur.

(b) $\delta_{t}(y) \geq 0$ because $\eta_{t}(y)^{2}-\widetilde{\xi}_{t}(y)^{2}=\sigma_{t}^{2}(y) \geq 0$.

(c) Substitution of $\delta_{t}+\widetilde{\xi}_{t}$ for $\eta_{t}$ in (11) gives

$$
\sigma_{t}^{2}(y)=2 \delta_{t}(y) \widetilde{\xi}_{t}(y)+\delta_{t}(y)^{2} .
$$

The rest is easy. For each $\epsilon>0$ define

$$
A_{t, \epsilon}:=\left\{y \in y: \widetilde{\xi}_{t}(y) \geq \frac{1}{2}, \sigma_{t}(y) \leq \epsilon\right\} .
$$

Integration of inequality (a) gives

$Q_{0} \sigma_{t}^{2}(y) \leq \frac{1}{2} t^{2} P_{0} \Delta_{0}^{2}+2 P_{0} r_{t}^{2}=O\left(t^{2}\right)+o\left(t^{2}\right) \leq C t^{2} \quad$ for some constant $C$,

which, together with (10), implies $Q_{0} A_{t, \epsilon} \rightarrow 1$ as $t \rightarrow 0$.

On the set $A_{t, \epsilon}$ equality (c) ensures that $\delta_{t}(y) \leq \sigma_{t}^{2}(y) \leq \epsilon \sigma_{t}(y)$; on $A_{t, \epsilon}^{c}$ the nonnegativity of $\delta_{t}$ and equality (c) give $\delta_{t}^{2} \leq \sigma_{t}^{2}$. Thus

$$
\begin{aligned}
Q_{0} \delta_{t}(y)^{2} & \leq \epsilon^{2} Q_{0} \sigma_{t}^{2}(y)\left\{y \in A_{t, \epsilon}\right\}+Q_{0} \sigma_{t}^{2}(y)\left\{y \notin A_{t, \epsilon}\right\} \\
& \leq \epsilon^{2} C t^{2}+\frac{1}{2} t^{2} Q_{0} \kappa_{y} \Delta_{0}^{2} A_{t, \epsilon}^{c}+2 Q_{0} \kappa_{y} r_{t}^{2} \quad \text { by (a). }
\end{aligned}
$$

The $Q_{0}$-integrability of $\kappa_{y} \Delta_{0}^{2}$ and the Dominated Convergence theorem imply $Q_{0} \kappa_{y} \Delta_{0}^{2} A_{t, \epsilon}^{c} \rightarrow 0$. It follows that $Q_{0} \delta_{t}^{2}=o\left(t^{2}\right)$.

Finally, what happens when $P_{t}$ is not dominated by $P_{0}$ ? The analysis for $\xi_{t}^{2}$, the density of the part of $P_{t}$ that is dominated by $P_{0}$, is the same as before. The image measure $S P_{t}^{\perp}$ has total mass of order $o\left(t^{2}\right)$, part of which gets absorbed into $Q_{t}^{\perp}$. The part of $S P_{t}^{\perp}$ that is dominated by $Q_{0}$ contributes an extra nonnegative term, $\gamma_{t}(y)$, to the density $d Q_{t}^{(a b s)} / d Q_{0}$. The $\eta_{t}^{2}(y)$ becomes $\kappa_{y} \xi_{t}^{2}(y)+\gamma_{t}(y)$. The extra term causes little trouble because

$$
\sqrt{\kappa_{y} \xi_{t}^{2}} \leq \eta_{t} \leq \sqrt{\kappa_{y} \xi_{t}^{2}}+\sqrt{\gamma_{t}} \quad \text { and } \quad Q_{0} \gamma_{t}=o\left(t^{2}\right)
$$

imsart-aos ver. 2011/11/15 file: pollard_april2012.tex date: May 25, 2018 
Proof of assertion (ii). Write $\mathbb{H}$ for the closed subspace of $L^{2}\left(P_{\theta}\right)$ consisting of (equivalence classes of) functions measurable with respect to the subsigma-field of $\mathcal{A}$ generated by $S$. Each member of $\mathbb{H}$ is of the form $f(S x)$ for some $f$ in $L^{2}\left(Q_{\theta}\right)$. The orthogonal projection of $\Delta_{\theta}$ onto $\mathbb{H}$ equals $\widetilde{\Delta}_{\theta}(S x)$. Thus

$$
\mathbb{I}_{\mathcal{P}}(\theta)=P_{\theta} \Delta_{\theta}(x)^{2}=P_{\theta} \widetilde{\Delta}_{\theta}(S x)^{2}+P_{\theta}\left[\Delta_{\theta}(x)-\widetilde{\Delta}_{\theta}(S x)\right]^{2} .
$$

The first term on the right-hand side equals $Q_{\theta}\left(\widetilde{\Delta}_{\theta}^{2}\right)=\mathbb{I}_{Q}$; the last term is zero if and only if $\Delta_{\theta}(x)=\widetilde{\Delta}_{\theta}(S x)$ a.s. $\left[P_{\theta}\right]$.

Acknowledgements. Many thanks to the referee for pointing out the connections with the work of Basu. In retrospect, it is a little surprising that I was unable to find a counterexample analogous to that of $K \& S$ in the volume (DasGupta, 2011) of Basu's selected works.

\section{References.}

BAsu, D. (1964). Recovery of ancillary information. Sankhyā: The Indian Journal of Statistics, Series A 26 3-16. Reprinted in Ghosh (1988).

BASU, D. (1975). Statistical information and likelihood. Sankhyā: The Indian Journal of Statistics, Series A 37 1-71. Reprinted in Ghosh (1988).

Bickel, P. J., Klahssen, C. A. J., Ritov, Y. and Wellner, J. A. (1993). Efficient and Adaptive Estimation for Semiparametric Models. Johns Hopkins University Press, Baltimore.

DasGupta, A. (2011). Selected Works of Debabrata Basu. Springer-Verlag.

Ghosh, J. K., ed. (1988). Statistical Information and Likelihood: A Collection of Critical Essays by Dr. D. Basu. Lecture Notes in Statistics 45. Springer-Verlag.

HÁJEK, J. (1972). Local asymptotic minimax and admissibility in estimation. In Proceedings of the Sixth Berkeley Symposium on Mathematical Statistics and Probability (L. Le Cam, J. Neyman and E. L. Scott, eds.) I 175-194. University of California Press, Berkeley.

Ibragimov, I. A. and Has'minskit, R. Z. (1981). Statistical Estimation: Asymptotic Theory. Springer, New York. (English translation from 1979 Russian edition).

Kagan, A. and Shepp, L. A. (2005). A sufficiency paradox: an insufficient statistic preserving the Fisher information. The American Statistician 59 54-56.

Le CAM, L. (1986). Asymptotic Methods in Statistical Decision Theory. Springer-Verlag, New York.

Le CAm, L. and YAng, G. L. (1988). On the preservation of local asymptotic normality under information loss. Annals of Statistics 16 483-520.

Le Cam, L. and Yang, G. L. (2000). Asymptotics in Statistics: Some Basic Concepts, 2nd ed. Springer-Verlag.

Lehmann, E. L. and Casella, G. (1998). Theory of Point Estimation, second ed. Springer.

Lehmann, E. L. and Romano, J. P. (2005). Testing Statistical Hypotheses, third ed. Springer.

Pitman, E. J. G. (1979). Some Basic Theory for Statistical Inference. Monographs on applied probability and statistics. Chapman and Hall.

imsart-aos ver. 2011/11/15 file: pollard_april2012.tex date: May 25, 2018 
VAN DER VAART, A. (1988). Statistical estimation in large parameter spaces. Center for Mathematics and Computer Science CWI Tract 44.

Statistics Department

YALE UNIVERSITY

New Haven, Connecticut

imsart-aos ver. 2011/11/15 file: pollard_april2012.tex date: May 25, 2018 